manifest itself. A simple quantitative measure of the resulting perturbation of the spectrum is the ratio of the amplitude of the central line to that of one of the hyperfine components. The broadening, and also, where it can be observed, the dipole-dipole splitting, depend on the inverse-cube of the separation, which can therefore be determined.

For a series of iminoxyl biradicals, in which the two paramagnetic centres are separated by structures of varying length, the separations so derived agree to about $1 \AA$ with those based on the concentration calibration for monoradicals. For oxyhaemoglobin bearing a spin label on a $\beta$-chain thiol group, the line-width parameter is the same as for the free label, whereas the same radical attached to methaemoglobin displays broadening, in consequence apparently of interaction with the highspin ferric haem iron atom. With two iodoacetate-based spin labels, one on each $\beta$-chain of oxyhaemoglobin, there is again no broadening, in accordance with the large separation of the two thiols in the molecule. If, on the other hand, a second label is introduced by way of a cyanuric fluoride derivative, which is thought to react with a histidine, interaction between this and the paramagnetic label on the thiol is observed, to an extent that indicates a separation of $25 \AA$. The size of the label makes this a somewhat vague parameter, and it is in any case at the upper distance limit of sensitivity. When the protein is denatured, however, the separation appears to decrease by 3 Å.

In an accompanying article (Kulikov et al., ibid., 40), the intramolecular interactions of a series of biradicals, mostly on rigid skeletons, are observed by the broadening of ESR signals from glassy low-temperature solutions, using the second moment of the spectrum as a criterion. The interaction falls off in the expected manner with separation, being very large at $12 \AA$, and barely measurable at $18 \AA$. The agreement between the separations, as calculated from the broadening and measured on molecular models, is remarkably good. Compounds containing more than two radicals, and even a copolymer of styrene, acrylic acid and an iminoxyl derivative, have also been examined in the same way. Interactions between spin labels on various groups of proteins are described; for example, the distance between the two reactive thiols needed for ATPase activity of myosin has been estimated at 14-18 $\AA$.

A similar approach to the measurement of distances between labelled molecules in a phospholipid bilayer has been explored by Marsh and Smith (Biochem. Biophys. Res. Commun., 49, 916; 1972). Spin-labelled cholestane introduced into bilayers at sufficient concentration shows large dipoledipole splitting when the magnetic field is perpendicular to the planes of the stacked bilayers. The separation of the labelled molecules in the plane is calculated from the splitting, and is found (not surprisingly) to vary with the composition of the phospholipid. In saturated phospholipid bilayers the spin-labelled species are close together, whereas the introduction of cholesterol drives them apart.

This general approach to the measurement of distances between groups capable of accepting a spin label may turn out to have considerable advantages over measurement of electronic excitation transfer. Whether it too will remain a technique of primarily decorative rather than utilitarian value in regard to real biological problems will remain to be seen. One has learned to read the signs in the lofty phrases, usually to be found at the ends of papers, regarding the promise and scope of whatever new method has just been trundled onto the launching pad.

\section{DESERT ANIMALS}

\section{Avoiding a Sulty Dief}

from our Animal Behaviour Correspondent

THE harsh environment of the desert poses many problems, such as those of combating heat and obtaining enough food and water, to animals like the kangaroo rats (Heteromydae) which live in the deserts of western North America. The possibility of obtaining food and water from such plants as can survive there is confounded by the fact that one of the adaptations shown by desert plants such as the saltbush (Atriplex) is to have a layer of salt-filled tissue at the surface of their leaves. This seems to help the sait-water balance of the plants but would make them dangerous eating for most animals because of the high concentration of salt. In one species of kangaroo rat, Dipodomys microps, this particular difficulty has been partially solved by the evolution of specialized teeth and behaviour which help to provide it with a year round supply of food.

G. J. Kenagy reports (Science, 178, 1094 ; 1972) that this species has broad, chisel-shaped lower incisors, unlike other species of kangaroo rat living in the same area, which have relatively narrow and rounded lower incisors. Chisel-shaped lower teeth enable it to strip off the salty outer layers of the leaves and obtain the inner portions of the leaves which are relatively salt-free.

D. microps holds a saltbush leaf in its forefeet and draws it over its lower teeth about ten times, so shaving off the outer layers of one side. It then turns the leaf over and does the same thing to the other side. Kenagy found that the discarded shavings are very high in electrolytes and are not eaten by the rats, whereas the sodium concentration of the inner tissue, which the rats eat, was only 3 per cent of that of the shavings. Furthermore, in a feeding experiment, Kenagy found that $D$. microps could survive for at least 16 days on a diet of nothing but saltbush leaves. A closely related species, $D$. merriami, would not eat these leaves and died within 2 days.

Saltbush leaves are available all year round, so that $D$. microps is provided with a predictable and continuous food supply. This minimizes competition with closely related species which subsist on seeds, but for whom the saltbush is like the water, water everywhere of the Ancient Mariner.

\title{
Homology of Fungal and Mammalian Protein
}

THE sequence of the enzyme glutamate dehydrogenase from bovine liver is known from the work of Smith and coworkers. These workers also showed that there is little divergence between this and the corresponding protein from chicken, only twenty-six substitutions being found in the 500 residues. Now Wootton, Chambers, Taylor and Fincham report in next Wednesday's Nature New Biology (January 10) their comparison of the NADP-dependent glutamate dehydrogenase of Neurospora crassa with the mammalian species.

Wootton et al. sequenced tryptic peptides and tried to index each of them on the sequence of the bovine liver enzyme. In general a unique bestmatch was found, with a vanishingly low statistical probability that it would have occurred by chance. There is a lysine in a position corresponding to the reactive lys-126 of the bovine sequence, and a general sequence homology around it extends the observation of a similar centre in the sequence of bovine glyceraldehyde 3-phosphate dehydrogenase.

Some Neurospora mutants were also examined, and it seems that a cluster of basic residues, his-lys-his, is somehow involved in stabilizing the enzymatically active conformation of the protein, for replacements in this position lead to anomalous activation behaviour. The existence of distinct homologies in many parts of the Neurospora and bovine glutamate dehydrogenase sequences is taken to signify a common evolutionary origin, rather than evolutionary convergence. 